Supporting Information

\title{
Quantitative drug release monitoring in tumors of living subjects by magnetic particle imaging nanocomposite
}

\author{
Xingjun Zhu ${ }^{1} \uparrow$, Jianfeng $\mathrm{Li}^{2} \uparrow$, Peng Peng ${ }^{1}$, Niloufar Hosseini Nassab ${ }^{1}$, Bryan Ronain \\ Smith ${ }^{1 *}$
}

1 Department of Radiology, Stanford University School of Medicine, Stanford, California 94305, USA

2 Department of Orthopaedic Surgery, Stanford University, Stanford, CA 94305, USA

*Corresponding Author: Bryan Ronain Smith: bryanrsmith2@gmail.com

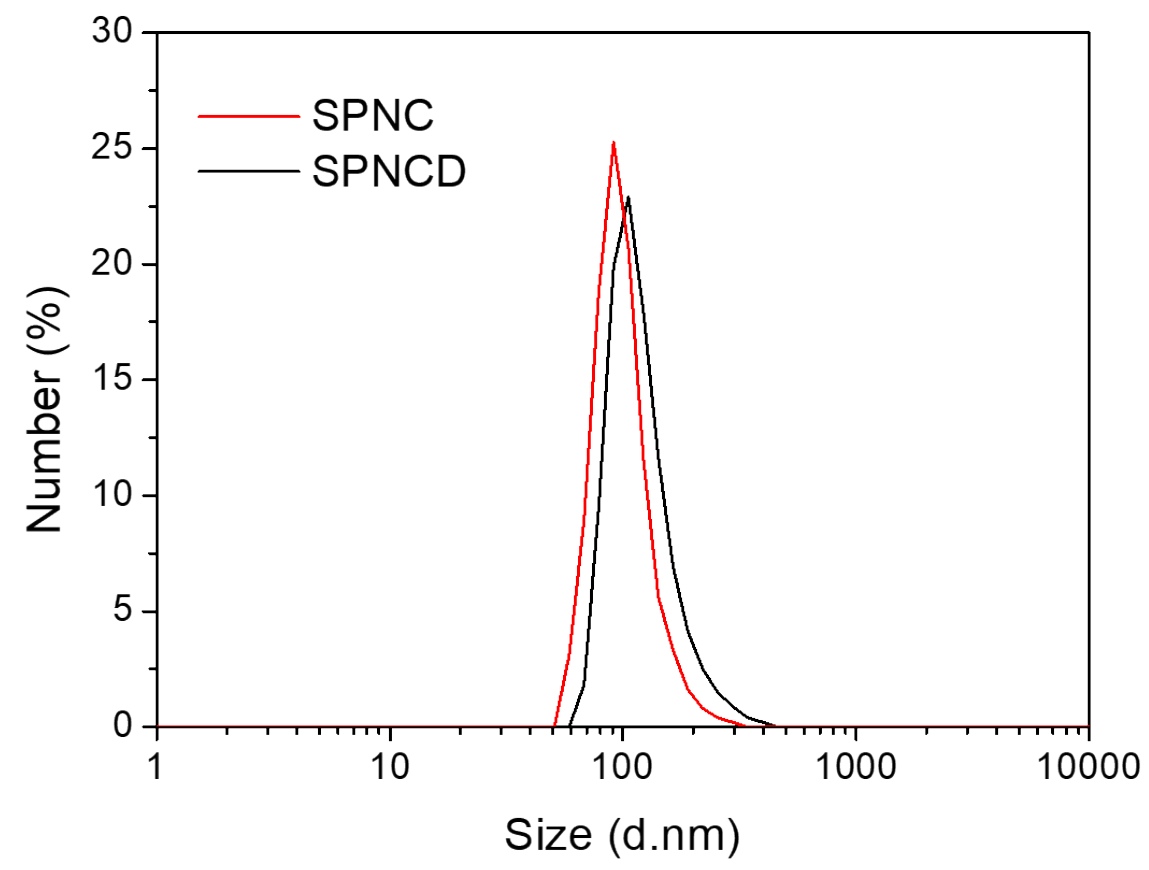

Figure S1. Dynamic light scattering measurement of the hydrodynamic size of SPNC and SPNCD nanocomposite. The diameter of SPNCD is slightly larger than SPNC because the loading of DOX leads to swelling of the nanostructure. 


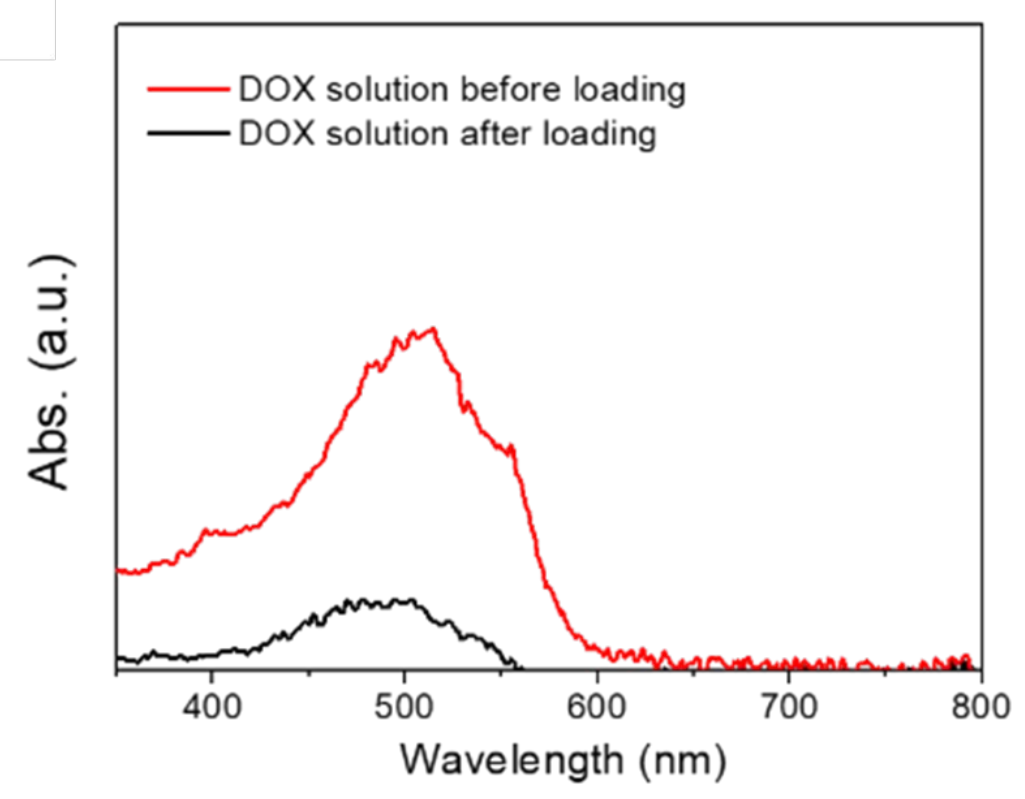

Figure S2. DOX is loaded into SPNC particles, generating SPNCD. Shown are UV-Vis absorption spectra of the DOX solution supernatant before and after incubation of SPNC. SPNC loaded with DOX (SPNCD) was separated by centrifugation and removed from the original DOX solution. The fluorescence intensity of the DOX solution after the removal of SPNCD is lower than the one before loading process, indicating that DOX is successfully loaded on SPNCD. 


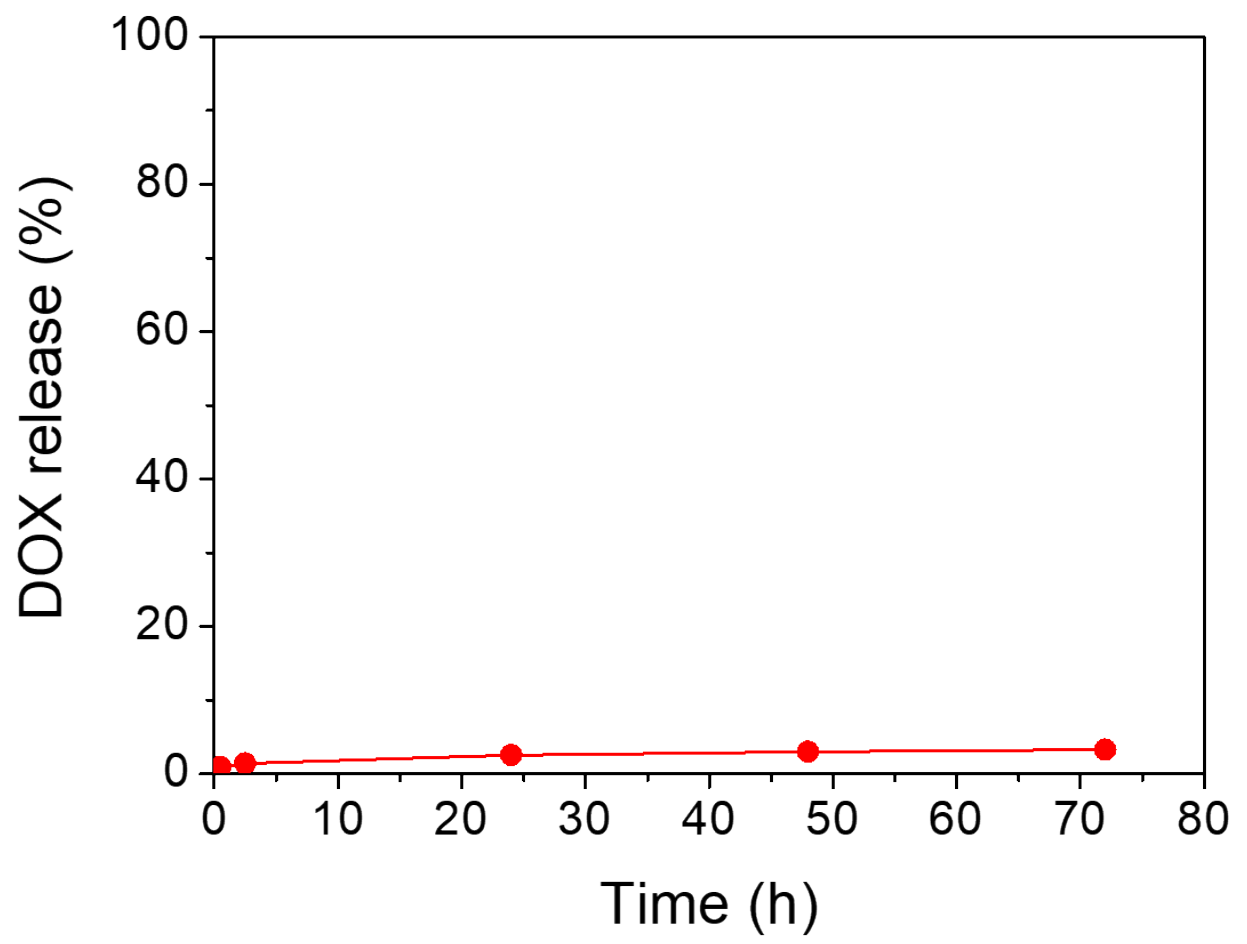

Figure S3. Time-dependent DOX release behavior of SPNCD in $\mathrm{pH}=7.4$ phosphate buffer. The DOX release in $\mathrm{pH}=7.4$ is less than $3 \%$ after $72 \mathrm{~h}$ of incubation. 


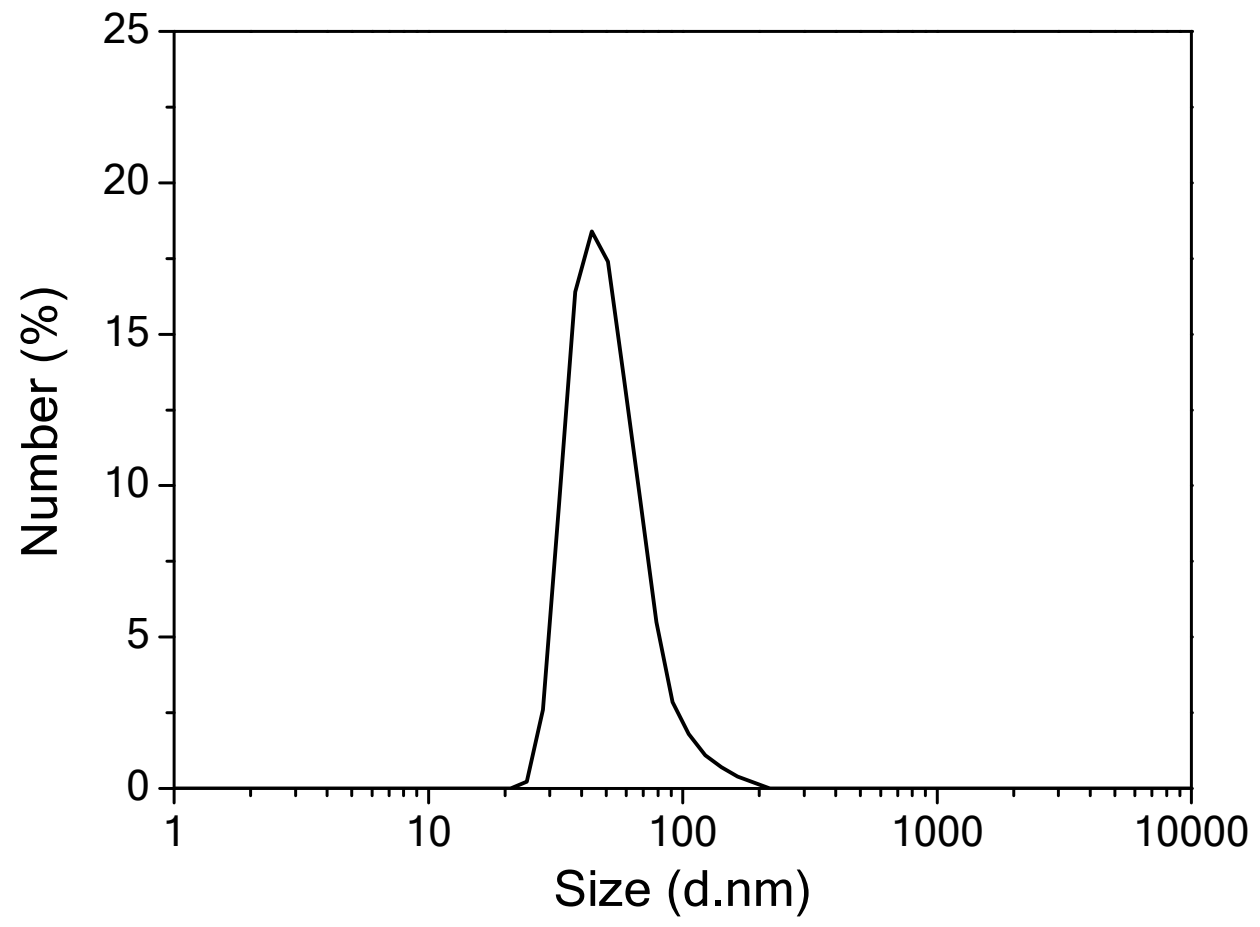

Figure S4. Dynamic light scattering measurement of the hydrodynamic size of SPNCD after incubation in $\mathrm{pH}=6.5$ phosphate buffer for $48 \mathrm{~h}$. The average size of the particles is $53.6 \mathrm{~nm}$, which is significantly smaller than the SPNCD before being incubated in $\mathrm{pH}=6.5$ phosphate buffer. These data confirm our SEM results, indicating that degradation of SPNCD occurs in acidic environments. 


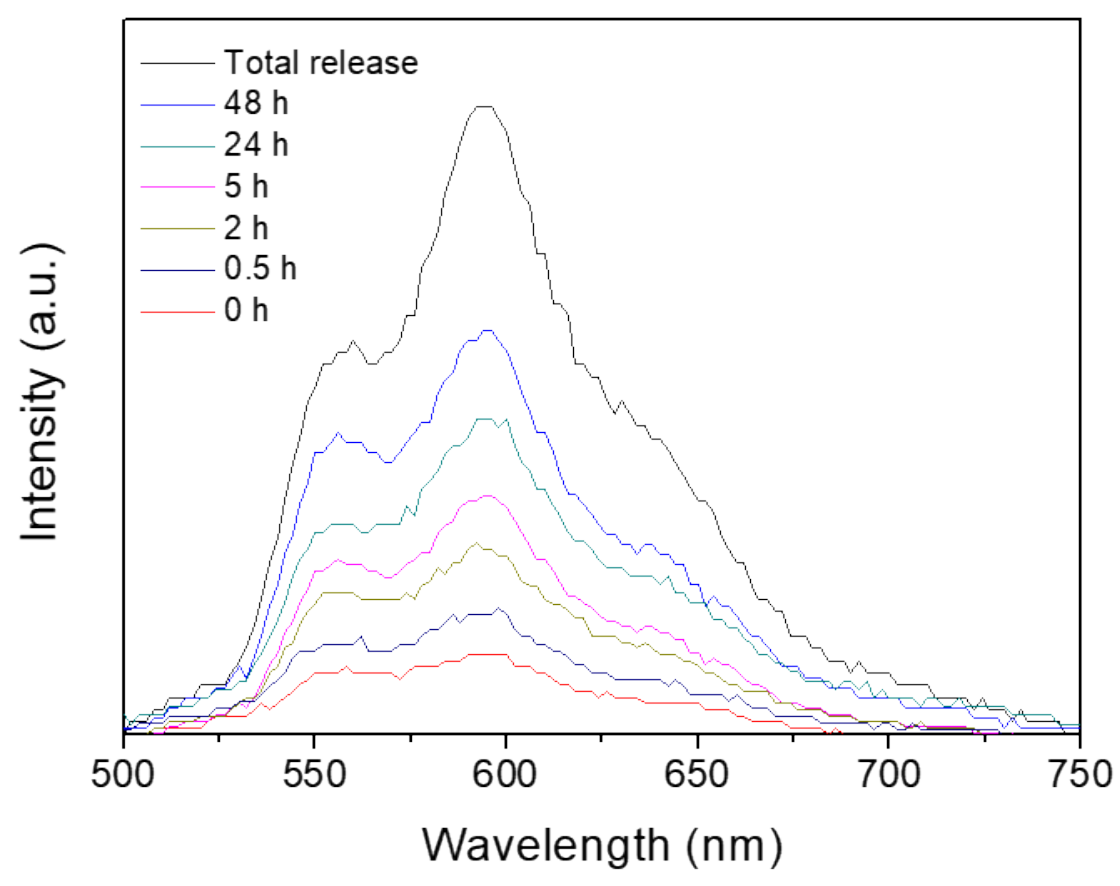

Figure S5. Fluorescence spectra of DOX detected from MDA-MB-231 cells incubated with SPNCD in buffer at different time-points. The 'Total release' spectrum was detected from the MDA-MB-231 cells incubated with pure DOX-containing buffer; the concentration of DOX is the same as that of DOX in SPNCD-containing buffer. 

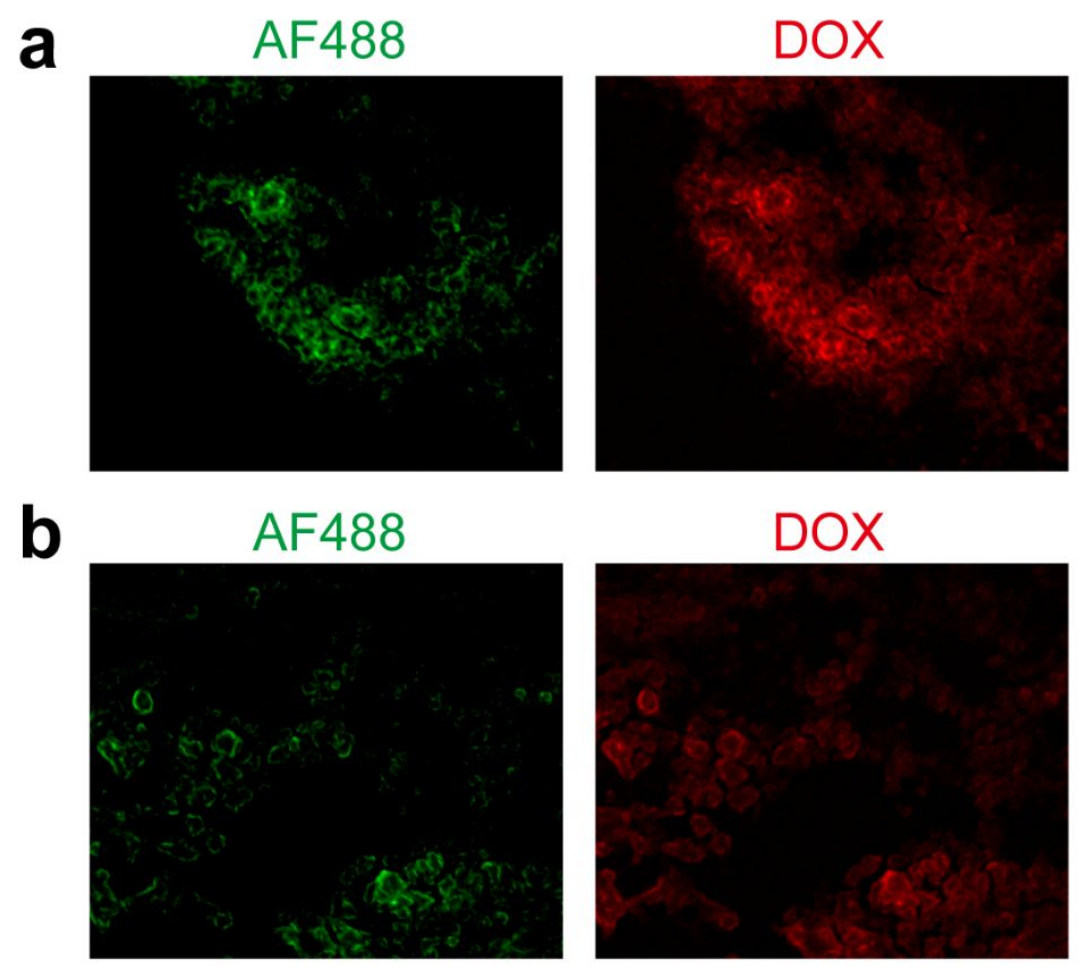

Figure S6. Separated fluorescent images of Annexin V-AF488 (apoptotic cells) and DOX in tumor sections of MDA-MB-231 tumor-bearing nude mice with intratumoral injection of a, DOX only, and b, SPNCD nanocomposites $48 \mathrm{~h}$ post-injection. The merged fluorescent images are shown in Figure $5 \mathrm{~d}$ of the main text. The DOX fluorescence intensity in the SPCND group appears lower than the intensity in the pure DOX group because some DOX remaining within the particles may continue to be quenched during release, compared to all DOX in the DOX group able to contribute to the fluorescence signal. 


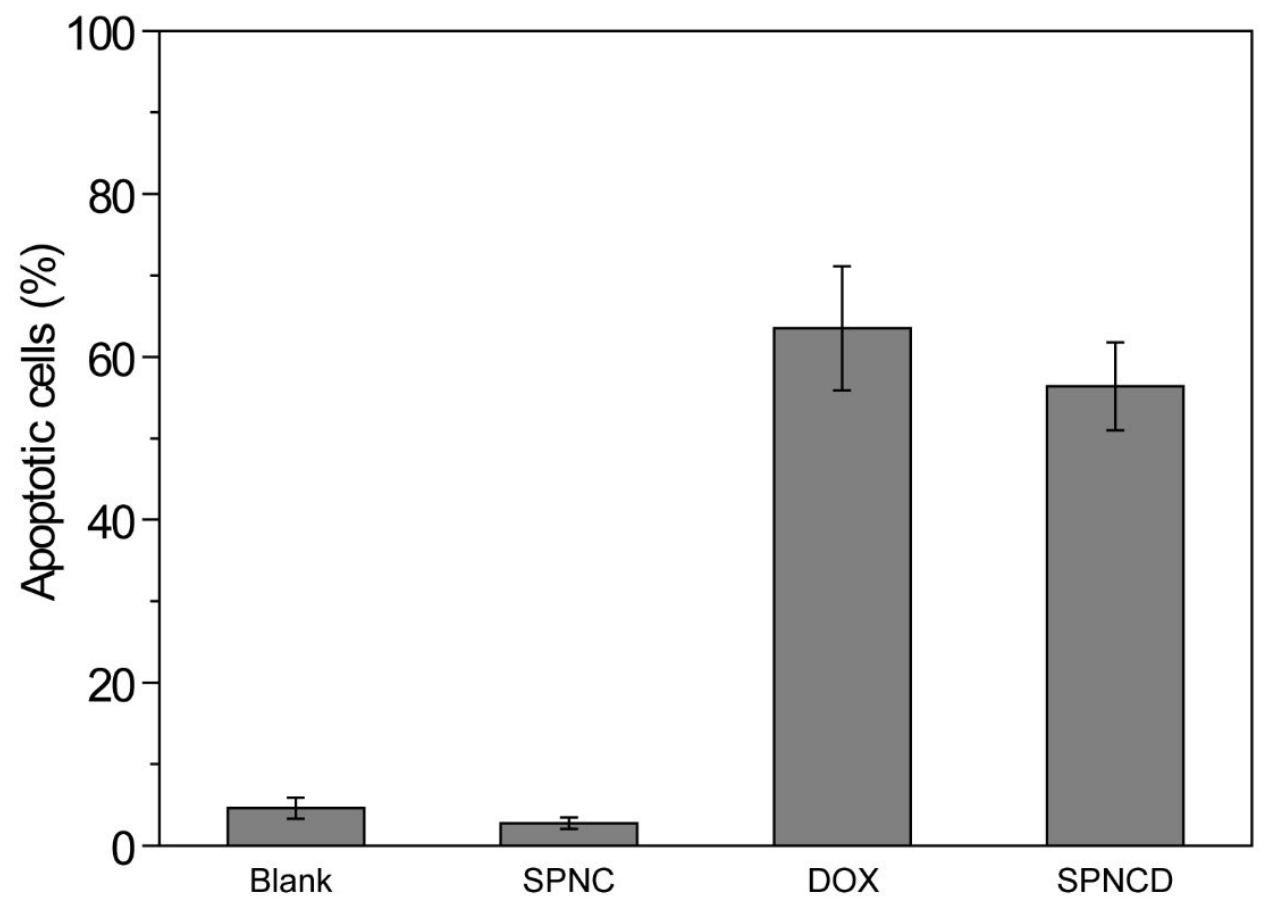

Figure S7. Semi-quantitative measure (as a \% of total cells) of apoptotic cells in tumor sections of MDA-MB-231 tumor-bearing nude mice with intratumoral injection of saline vehicle alone (Blank), SPNC, DOX only, and SPNCD.
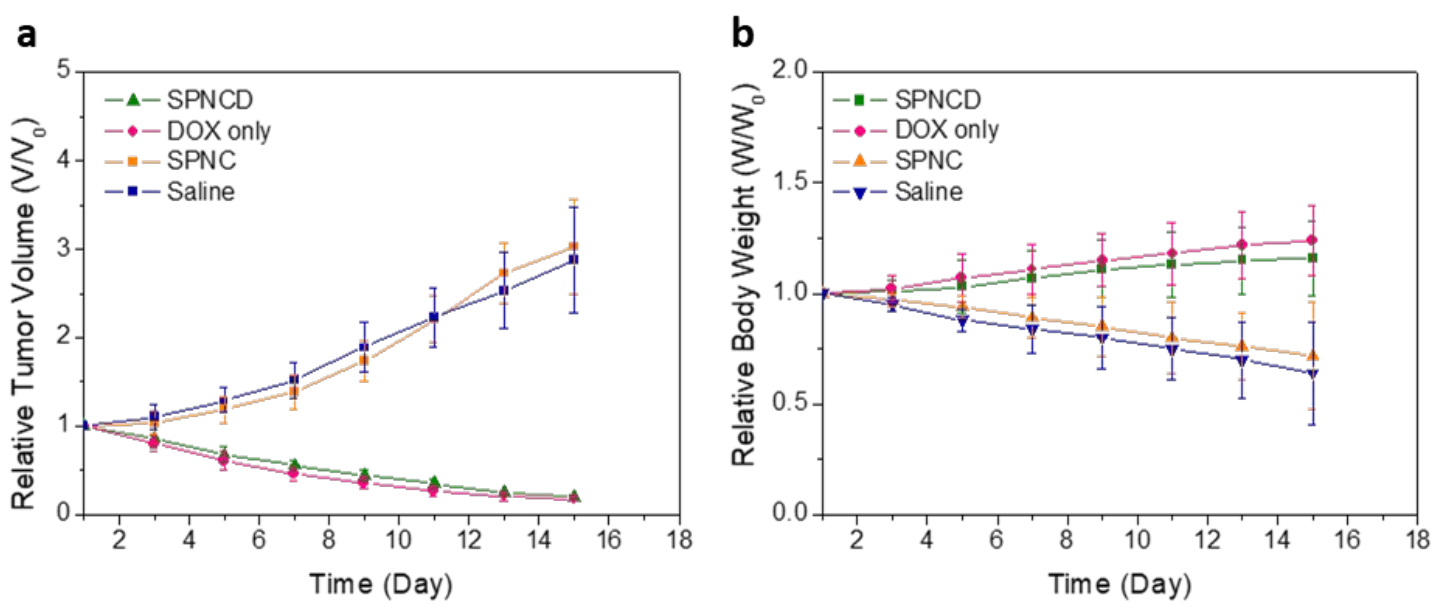

Figure S8. a, Growth of tumors in different groups of mice $(\mathrm{N}=3)$ after treatment. The relative tumor volumes were normalized to their initial sizes. Average values of the tumor volume of each group were based on the measurement of the tumors in three mice/group. Error bars are presented as S.E.M. b, Body weights in different groups of mice $(\mathrm{N}=3)$ after 
treatment. The relative body weights were normalized to their initial weights. The average values of the body weights of each group were based on the measurement of weights in three mice/group. Error bars are presented as S.E.M. 